\title{
Effects of lysine-acetylsalicylate (LAS) treatment in nasal polyposis: two controlled long term prospective follow up studies
}

\author{
E Nucera, D Schiavino, A Milani, M Del Ninno, C Misuraca, A Buonomo, \\ C D'Ambrosio, G Paludetti, G Patriarca
}

Nasal polyposis is a multifactorial disease with a complex and still not completely understood pathogenesis. In more than one third of cases it is associated with intolerance to acetylsalicylic acid (aspirin, ASA) or to other non-steroidal anti-inflammatory drugs (NSAIDs). ${ }^{1}$ In as many as $20 \%$ of cases nasal polyposis is also associated with the presence of bronchial asthma and/or rhinitis, configuring the socalled ASA triad or aspirin disease. ${ }^{2}$

Nasal polyps may benefit from medical treatment (corticosteroids) and surgery, ${ }^{3}$ but they frequently relapse soon after surgery ${ }^{4-9}$ with significant morbidity and high social and medical costs. Unfortunately, the effect of treatment with steroids is also often temporary. $^{3}$ 10-16

In the last two decades it has been observed that, in aspirin sensitive patients, oral aspirin desensitisation (followed by long term aspirin treatment) often results in an improvement in the clinical course of nasal polyposis. ${ }^{17-21} \mathrm{We}$ have shown that aspirin sensitive patients with nasal polyposis have a higher rate of positive nasal provocation tests (rhinomanometric measure of nasal airflow reduction after exposure to the drug) with lysineacetylsalicylate (LAS) than aspirin sensitive patients without nasal polyps. ${ }^{22-24}$ Moreover, LAS has been found to have an in vitro non-specific antiproliferative, dose dependent effect on the growth of fibroblasts of both nasal polyps and normal skin. ${ }^{23}$ We have shown that long term topical (endonasal) treatment with LAS prevents the recurrence of nasal polyps after polypectomy with satisfactory results. ${ }^{24}$

In this paper we present and discuss the definitive results obtained in two controlled long term prospective follow up studies dealing with the evaluation of relapse rates in nasal polyposis.

The first is a six year follow up study of patients with nasal polyps who underwent long term intranasal LAS treatment after surgical polypectomy in comparison with matched controls (patients who underwent simple polypectomy with no further treatment). The cumulative percentage of patients in whom polyps did not recur during the follow up period was evaluated by the life table method and compared with that of controls using a $\chi^{2}$ log rank test. ${ }^{25}$ This first controlled follow up will be referred to as "SP-LAS" (surgical polypectomy + LAS treatment). In the second controlled study a group of patients suffering from nasal polyposis (in whom surgery was indicated) received a "medical" polypectomy (corticosteroids) followed by long term topical LAS treatment instead of surgery. A successive three year prospective follow up was completed. This second follow up study will be referred to as "MP-LAS" (medical polypectomy + LAS treatment).

\section{Methods}

SELECTION OF PATIENTS

In both studies consecutive patients suffering from nasal polyposis were followed as outpatients at the Department of Allergology of Policlinic A Gemelli, Rome, Italy. In all cases a diagnosis of nasal polyposis was established by means of clinical criteria (ENT examination including rhinoscopy, standard skull radiographs, and computed tomographic (CT) scan of the nasal and paranasal cavities). The severity of symptoms (impaired quality of life despite appropriate medical treatment, chronic nasal congestion, recurrent infections, bone erosion, mucoceles, worsening asthma, or other complications) indicated surgery in all patients.

According to the principles of the Helsinki declaration, informed consent to the entire protocol was obtained from each patient.

All subjects underwent a complete allergological evaluation including skin prick tests with the commonest inhaled allergens (Graminae, Parietaria, olive, cat, dust mite, Aspergillus, Penicillium, Alternaria; ALK-Abello, Madrid, Spain). Serum total (PRIST, Pharmacia) and specific (RAST, Pharmacia) IgE determinations were also performed.

A nasal provocation test (NPT) with LAS was performed in all subjects. NPT measures, by means of anterior rhinomanometry, ${ }^{26-28}$ the percentage decrease in nasal airflow following the inhalation of increasing doses of the specific allergen. The NPT response was considered positive if inhalation of the specific allergen resulted in a reduction in airflow of more than $40 \%$ of the basal value. In patients with a positive skin prick or RAST test an NPT was also performed, inhaling the specific allergen to confirm the occurrence of any hypersensitivity. After measuring basal (untreated) nasal airflow, non-specific hyperreactivity of the nasal mucosa was excluded by measuring nasal airflow before and 10 and 20 minutes after inhaling saline $(2 \mathrm{ml}, 0.9 \%)$. Thereafter, an initial dose of $20 \mu \mathrm{g}$ LAS in $2 \mathrm{ml}$ saline was administered. Nasal airflow was evaluated 10 and 20 minutes after the allergen challenge. Where the NPT response was negative, $200 \mu \mathrm{g}$ LAS and, if further negative, 2 and $4 \mathrm{mg}$ LAS 
were administered, with an interval of 20 minutes, recording nasal airflow 10 and 20 minutes after inhalation. Nasal airflow was also determined 60 and 360 minutes after the positive response to identify any delayed response.

In both studies a complete ENT examination and a CT scan of the skull were performed every 6-12 months in all patients admitted to the follow up.

SP-LAS STUDY

Seventy six consecutive patients with nasal polyps (41 men, 35 women) aged 14-71 years were enrolled over an eight year period (198895). According to ENT indications, all patients underwent surgical ablation (Pietrantoni-De Lima technique). About one month after surgery inhalation treatment with LAS (six times/week) was started. The initial dose corresponded to $20 \mu \mathrm{g}$ aspirin, progressively increasing to a maintenance dose corresponding to $4 \mathrm{mg}$ aspirin six times/week.

MP-LAS STUDY

Forty nine patients ( 21 men, 28 women) aged 19-71 with nasal polyps in whom surgery was indicated were admitted to the follow up study during the period 1995-8. In all subjects a simple "medical polypectomy" was performed, administering $40 \mathrm{mg}$ triamcinolone retard intramuscularly every 10 days (three administrations, total dose $120 \mathrm{mg}$ ). Such a treatment achieves a decrease (at least temporary) in polyp volume and facilitates penetration of LAS into the rhinosinusal cavities. Thereafter, increasing doses of LAS were administered by inhalation according to the protocol described for the SP-LAS study.

CONTROL SUBJECTS

Both the follow up studies shared the same control population consisting of 191 age matched subjects suffering from nasal polyposis (101 men, 90 women, age 16-70) who had previously undergone surgical polypectomy with no further medical treatment. This group of patients may represent a somewhat "improper" control population for the MP-LAS study which involves patients who did not undergo any surgical polypectomy. However, the MP-LAS prospective study cannot have a "natural" control group since obvious ethical considerations prevent follow up without any further treatment (besides initial corticosteroids) in a population of patients suffering from severe nasal polyps in whom surgery is indicated.

STATISTICAL ANALYSIS

In both the follow up studies the rates of recurrence of nasal polyps were evaluated by the life table method. ${ }^{25}$ This statistical tool is able to include in the analysis data on subjects abandoning the study at any time, evaluating at any considered time interval the actual number of patients still at risk. The difference in rates of recurrence of the investigated patients and the control subjects were evaluated by the log rank test $\mathrm{t}^{25}$ which gives a "relative" rate of recurrence for each investigated population. Such a figure

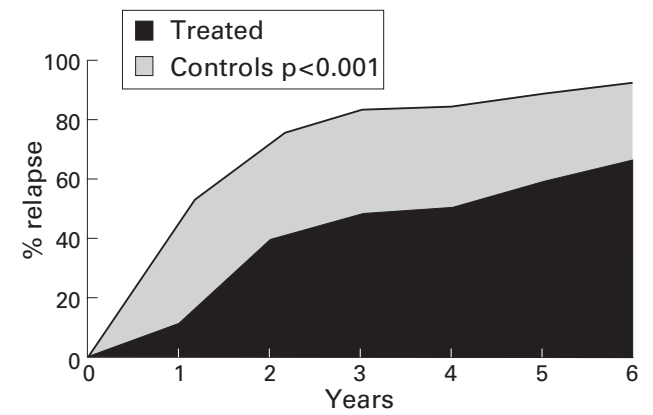

Figure 1 SP-LAS follow up results: cumulative percentage relapse of nasal polyposis after surgery (life table method).

represents the ratio between the average recurrence rate of the patients and the overall (investigated subjects and controls) averaged rate of recurrence of polyps (that is arbitrarily set at 1.00). This permits an easy and global quantitative comparison between different populations and the computing of appropriate $\chi^{2}$ statistics.

\section{Results}

SP-LAS STUDY

Thirty eight $(50 \%)$ of the 76 enrolled patients had aspirin sensitivity (mainly with asthmatic symptoms) and in 28 subjects $(36.8 \%)$ the complete aspirin triad syndrome was diagnosed. A moderate to severe degree of anosmia was present in 62 patients $(81.6 \%)$, in $72.6 \%$ of cases associated with aspirin intolerance.

Thirty patients $(39.5 \%)$ had positive skin prick tests (19 to Dermatophagoides, eight to Graminae, and three to Parietaria); these subjects had a history of rhinitis (19 patients) or asthma (11 subjects). However, a true allergic reaction (positive RAST and/or specific NPT) was detected in only 25 subjects $(32.9 \%)$

LAS treatment was well tolerated by all patients and no adverse reactions were observed. LAS-NPT was positive in 24 cases $(31.6 \%)$.

The results of the SP-LAS prospective study are summarised in fig 1 which shows the cumulative percentages (life table method) of patients in whom there was no recurrence of nasal polyps after polypectomy over the six year follow up period. A highly significant statistical difference was seen between the relapse rates of LAS treated subjects and controls $\left(\chi^{2} 13.4 ; \mathrm{df}\right.$ $=1 ; \mathrm{p}<0.001, \log$ rank test). The relative relapse rate of the controls (1.40) was nearly twice that of the treated patients $(0.78)$. The percentage of controls in whom nasal polyps had not recurred was $48.7 \%$ at one year, declining to $15.2 \%$ after three years and to $6.5 \%$ six years after surgery. Conversely, $93.1 \%$ of patients treated with LAS were relapse-free at one year, $55.1 \%$ at three years, and nearly $35 \%$ six years after surgery.

No statistical difference in the cumulative rates of recurrence were found between aspirin tolerant and aspirin sensitive treated patients and controls. 


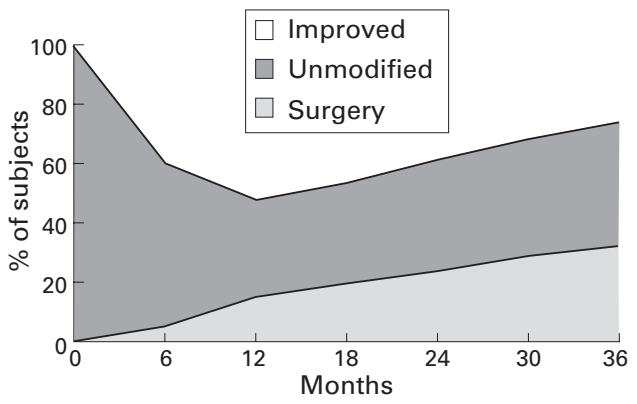

Figure 2 MP-LAS follow up results: cumulative percentage of subjects with polyposis after "medical" polypectomy (corticosteroids) followed by topical LAS treatment (life table method).

MP-LAS STUDY

Twenty five of the 49 investigated patients $(51.0 \%)$ were aspirin sensitive (mainly with asthmatic symptoms; only three patients had urticaria-angioedema). In 14 cases $(28.6 \%)$ the complete clinical picture of the aspirin triad was evident. Most patients $(87.8 \%)$ had anosmia (in $72.1 \%$ of cases associated with sensitivity to aspirin).

Twenty patients $(40.8 \%)$ had positive skin tests (13 to Dermatophagoides, five to Graminae and two to Parietaria); 11 had rhinitis and nine had symptoms of asthma. However, a true allergic reaction (positive RAST and/or specific NPT) was detected in only 10 subjects (20.4\%).

Treatment with LAS and NPT were well tolerated by all patients and no adverse reactions were observed. LAS-NPT was positive in 16 cases $(32.6 \%)$.

Figure 2 summarises the results obtained in the three year MP-LAS follow up. The percentage of patients whose symptoms and clinical picture improved after LAS treatment (with no surgical polypectomy) was $42.4 \%$ after six months, $54.3 \%$ at one year, and was nearly $30 \%$ after three years. These figures are not statistically different (log rank test) from the relapse-free rates obtained in controls (who did undergo surgical polypectomy), which did not reach $50 \%$ one year after surgery and were $15 \%$ three years after polypectomy (fig 1). As already stated, such a comparison may be somehow improper and it should be regarded just as indicative.

About one third of the patients treated with LAS showed no change in nasal polyps after three years of treatment (fig 2). The cumulative percentage of patients in whom the disease worsened and who therefore had to be treated surgically was just $3.8 \%$ in the first six months, rising to $13.1 \%$ after one year and to $32 \%$ at the end of the three year follow up. These last figures appear to be surprisingly low in a population of patients suffering from severe nasal polyps requiring surgery.

As in the SP-LAS study, no statistical difference was observed in the rates of recurrence of nasal polyps between aspirin tolerant and aspirin sensitive subjects.

\section{Discussion}

Our results indicate that long term topical treatment with LAS may be clinically effective in nasal polyposis. The potential effectiveness of topical LAS in the prevention of recurrence of nasal polyps after surgery has already been reported $^{2429}$ and our preliminary results have substantially been confirmed by other authors. ${ }^{30}$ The present study provides further evidence of the effectiveness of LAS treatment following surgery in patients with nasal polyposis, and demonstrates also the favourable effects of LAS in patients who did not have surgery even though surgery was indicated.

The favourable effect of LAS in nasal polyposis is probably due to its non-specific anti-inflammatory properties rather than to desensitisation since LAS is highly effective in both aspirin tolerant and aspirin sensitive patients. These findings are confirmed by a previous report ${ }^{23}$ of the inhibitory effect of LAS on the growth of cultures of nasal polyps on normal skin fibroblasts from aspirin tolerant and aspirin sensitive patients. It was dose dependent, non-specific, and "cytostatic" since cell viability was always as high as $85 \%$.

Long term topical treatment with LAS is almost free of side effects and it may therefore be used in subjects known to be at risk, such as those suffering from the aspirin triad.

In conclusion, in patients with nasal polyposis a medical approach should always be performed before surgery which has, in most cases only, transient results.

1 Slavin RG, Linford P, Friedman WH. Sinusitis and bronchial asthma. 7 Allergy Clin Immunol 1982;69:102-9.

2 Samter M, Beers RF. Concerning the nature of intolerance to aspirin. F Allergy 1967;40:281-6.

3 Lildholdt T, Fogstrup J, Gammelgaard N, et al. Surgical versus medical treatment of nasal polyps. Acta Otolaryngol 1988;105:140-3.

4 Blumstein GI, Tuft L. Allergy treatment in recurrent nasal polyposis. Am Med Sci 1957;234:269-74.

5 Brown BL, Harner SCG, Van Dellen RG. Nasal polypectomy in patients with asthma and sensitivity to aspirin. Arch Otolaryngol 1979;105:413-6.

6 Virolainen E, Puhakka H. The effect of intranasal beclomethasone dipropionate on the recurrence of nasal polyps after ethmoidectomy. Rhinology 1980;18:9-18.

7 Jaennti-Alanko S, Holopainen E, Malberg H. Recurrence of nasal polyps after surgical treatment. Rhinology 1989;8(S): 59-64.

8 Wigand ME, Hosemann W. Microsurgical treatment of recurrent nasal polyposis. Rhinology 1989;8:25-30.

9 Friedman WH, Katsantonis GP. Intranasal and transnasal ethmoidectomy: a 20-year experience. Laryngoscope 1990; 100:343-8.

10 Ruhno J, Andersson B, Denburg J, et al. A double-blind comparison of intranasal budesonide with placebo for nasal polyposis. F Allergy Clin Immunol 1990;86:946-53.

11 Kennedy DW. Prognostic factors, outcomes and staging in ethmoid sinus surgery. Laryngoscope 1992;57(Suppl) 1-18.

12 Braun JJ, Haas F, Conraux C. Polyposis of the nasal sinuses: epidemiology and clinical aspects of 350 cases. Treatment and results with a follow-up over 5 years on 93 cases. Ann Otolaryngol Chir Cervicofac 1992;109:189-99.

13 Van Camp C, Clement PAR. Results of oral steroid treatment in nasal polyposis. Rhinology 1994;32:5-10.

14 Lildholdt T, Rundcrantz H, Linqvist N. Efficacy of topical corticosteroid powder for nasal polyposis: a double-blind, placebo-controlled study of budesonide. Clin Otolaryngol 1969;90:110-5.

15 Mygind N. Effects of corticosteroideal therapy in nonallergic rhinosinusitis. Acta Otorhinolaryngol 1996;116:1649.

16 Holmberg K, Karlsson G. Nasal polyps and medical or surgical management. Clin Experim Allergy 1996;26(Suppl 3):23-30.

17 Stevenson DD, Simon RA, Mathison DA. Aspirin-sensitive asthma: tolerance to aspirin after positive oral aspirin challenges. F Allergy Clin Immunol 1980;66:82-8.

18 Lumry WR, Curd JG, Zeiger RS, et al. Aspirin-sensitive rhinosinusitis: the clinical syndrome and effects of aspirin administration. F Allergy Clin Immunol 1983;71:580-7.

19 Kietzman H, Brasch J, Rahmel U. Azetylsauretoleranzinduktion zur therapie der chronisch polyposen
rhinosinusitis bei bekannten intoleranzsyndrom. Allergolorinosinusitis bei beka 
20 Sweet JA, Stevenson DD, Simon RA, et al. Long term effects of aspirin desensitization treatment for aspirin sensitive rhinosinusitis asthma. $f$ Allergy Clin Immuno 1990;86:59-65

21 Stevenson DD, Hankammer MA, Mathisin DA, et al. Long term ASA desensitization-treatment of aspirin sensitive asthmatic patients: clinical outcome studies. F Allergy Clin Immunol 1996;98:751-8.

22 Patriarca G, Nucera E, Di Rienzo V, et al. Nasal provocation test with lysine acetyl-salicylate (LAS) in aspirin-sensitive patients. Ann Allergy 1991;67:60-5.

23 Bruzzese N, Sica G, Iacopino F, et al. Growth inhibition of fibroblasts from nasal polyps and normal skin by lysine acetyl-salicylate. Allergy 1998;53:431-4.

24 Patriarca G, Bellioni P, Nucera E, et al Intranasal treatment with lysine acetyl-salicylate in patients with nasal polyposis. Ann Allergy 1991;67:588-92.
25 Peto R, Pike MC, Armitage P. Design and analysis of randomized clinical trials requiring prolonged observation of omized clinical trials requiring prolonged

26 Taylor G, Shivalear PR. Changes in nasal airways resistance on antigenic challenge in allergic rhinitis. Clin Allergy 1971; on antigeni.

27 Girard JP, Horvat L, Heimliche M. Immunopathologie de la thinite allergique. Tests de provocation nasaux. Rev Franc Allergol 1974;14:175-9.

28 Borum P. Nasal methacoline challenge: a test for the measurement of nasal reactivity. F Allergy Clin Immunol 1979;63:253-6.

29 Patriarca G, Schiavino D, Nucera E, et al. Prevention of relapse in nasal polyposis. Lancet 1991;337:1488.

30 Scadding GK, Hassab M, Darby YC, et al. Intranasal lysineaspirin in recurrent nasal polyposis. Clin Otolaryngol 1995; 20:561-3. 